\title{
Sobolev Extension on $L^{p}$-quasidisks
}

\section{Zheng Zhu'}

Received: 3 June 2021 / Accepted: 11 August 2021 / Published online: 10 September 2021

(C) The Author(s) 2021

\begin{abstract}
In this paper, we study the Sobolev extension property of $L^{p}$-quasidisks which are the generalizations of classical quasidisks. After that, we also find some applications of this property.
\end{abstract}

Keywords Homeomorphism of finite distortion - Sobolev extension domains ·

$L^{p}$-quasidisks

Mathematics Subject Classification (2010) 30C65 - 46E35

\section{Introduction}

Let $\Omega \subset \mathbb{R}^{2}$ be a domain. A homeomorphism $h: \Omega \rightarrow \mathbb{R}^{2}$ is said to be quasiconformal if $h \in W_{\mathrm{loc}}^{1,2}\left(\Omega, \mathbb{R}^{2}\right)$ and the inequality

$$
|D h(z)|^{2} \leq K J_{h}(z)
$$

holds for almost every $z \in \Omega$ with a constant $K \in[1, \infty)$ that is independent of $z$. A bounded simply connected domain $\Omega \subset \mathbb{R}^{2}$ is called a quasidisk if there exists a global quasiconformal mapping $h: \mathbb{R}^{2} \stackrel{\text { onto }}{\longrightarrow} \mathbb{R}^{2}$ with $h(\Omega)=\mathbb{D}$. Quasidisks have lots of nice geometrical and potential properties, see the textbook by Gehring and Hag [13] and references therein. For example, a bounded simply connected planar domain is a quasidisk if and only if it is a uniform domain. Uniform domains were first introduced by Martio and Sarvas [30]. A domain $\Omega \subset \mathbb{R}^{2}$ is called uniform, if there exists a constant $C<\infty$ such that for every $z_{1}, z_{2} \in \Omega$ there is a curve $\gamma_{z_{1}, z_{2}} \subset \Omega$ with endpoints $z_{1}$ and $z_{2}$ and

$$
l\left(\gamma_{z_{1}, z_{2}}\right) \leq C d\left(z_{1}, z_{2}\right)
$$

and for all $z \in \gamma_{z_{1}, z_{2}}$ the inequality

$$
\min \left\{l\left(\gamma_{z_{1}, z}\right), l\left(\gamma_{z, z_{2}}\right)\right\} \leq C d\left(z, \mathbb{R}^{2} \backslash \Omega\right)
$$

The author was supported by the Academy of Finland (project No. 323960). The author thanks Prof. P. Koskela and Prof. J. Onninen for some interesting discussion and Prof. T. Kilpeläinen for improving the writing of the paper.

Zheng Zhu

zheng.z.zhu@jyu.fi

1 Department of Mathematics and Statistics, University of Jyväskylä, P.O.Box 35 (MaD) FI-40014, Jyväskylä, Finland 
holds, where $\gamma_{z_{1}, z}$ and $\gamma_{z, z_{2}}$ mean the subcurves of $\gamma_{z_{1}, z_{2}}$ from $z_{1}$ to $z$ and from $z$ to $z_{2}$ respectively. By Jones' result in [24], uniform domains hence quasidisks are $\operatorname{Sobolev}(p, p)$ extension domains for arbitrary $1 \leq p \leq \infty$. We say $\Omega \subset \mathbb{R}^{2}$ is a Sobolev $(p, q)$-extension domain for $1 \leq q \leq p \leq \infty$ if for every $u \in W^{1, p}(\Omega)$, there exists a function $E(u) \in$ $W^{1, q}\left(\mathbb{R}^{2}\right)$ with $\left.E(u)\right|_{\Omega} \equiv u$ and

$$
\|E(u)\|_{W^{1, q}\left(\mathbb{R}^{2}\right)} \leq C\|u\|_{W^{1, p}(\Omega)}
$$

with a positive constant $C$ that is independent of $u$.

There are many planar simply connected domains that are not quasidisks, for example inward and outward cuspidal domains, see [16, 18, 21, 22, 25-27]. Hence, it is natural to study generalizations of quasiconformal mappings. For instance, we concentrate on homeomorphisms of finite distortion here. A homeomorphism $h: \Omega \rightarrow \mathbb{R}^{2}$ is said to be a homeomorphism of finite distortion if $h \in W_{\text {loc }}^{1,1}\left(\Omega, \mathbb{R}^{2}\right)$ and the inequality

$$
|D h(z)|^{2} \leq K(z) J_{h}(z)
$$

holds for almost every $z \in \Omega$ with a measurable function $K(z) \in[1, \infty)$. For a homeomorphism of finite distortion $h$, we denote $K_{h}$ to be the optimal distortion function for (1.2) which will be defined in (2.3). If $K_{h} \in L^{\infty}(\Omega)$, then $h$ is quasiconformal. The inverse of a quasiconformal mapping is still quasiconformal. However, if we relax the regularity of the distortion function from essentially boundedness to some weaker condition, we cannot hope that the distortion of the inverse will attain the same regularity, see [17, 18]. Motivated by the Sobolev extension property of quasidisks, we can pose two following interesting problems.

(1) What is the best Sobolev extension property of domains which can be mapped onto the unit disk by homeomorphisms of finite distortion whose distortion functions satisfy some regularity?

(2) What is the best Sobolev extension property of domains which are images of the unit disk under homeomorphisms of finite distortion whose distortion functions satisfy some regularity?

In this paper, we mainly concentrate on the first problem with the condition that distortion functions are locally $L^{p}$-integrable for $1 \leq p<\infty$. Under this condition, the corresponding bounded domains are called $L^{p}$-quasidisks. The terminology $L^{p}$-quasidisk was firstly introduced in [22] by the author with Iwaniec and Onninen. In that paper, we gave characterizations of polynomial cuspidal domains which are $L^{p}$-quasidisks simultaneously. In [22], we showed that every bounded domain with a rectifiable boundary is a $L^{1}$-quasidisk. Hence, locally $L^{1}$-integrablity of distortion function is not enough to permit Sobolev extension.

Theorem 1.1 There exists a $L^{1}$-quasidisk which is not a Sobolev $(p, q)$-extension domain for any $1 \leq q \leq p<\infty$.

The main result in [22] tells us that for a fixed $1<p<\infty$, polynomial cuspidal domains with a relatively not very sharp singularities are $L^{p}$-quasidisks. Hence, for $1<p<\infty$, we can not hope every $L^{p}$-quasidisk is a Sobolev $(k, k)$-extension domain for arbitrary $1 \leq k<\infty$, see [32-35]. The following theorem will imply the Sobolev extension property of $L^{p}$-quasidisks as a special case. For a simply connected bounded domain $\Omega \subset \mathbb{R}^{2}$, the complementary set $\mathbb{R}^{2} \backslash \bar{\Omega}$ is also a domain.

Theorem 1.2 Let $\Omega \subset \mathbb{R}^{2}$ be a bounded simply connected domain and $0<R<\infty$ such that $\bar{\Omega} \subset B(0, R)$. If there exists a homeomorphism of finite distortion $h: \mathbb{R}^{2} \stackrel{\text { onto }}{\longrightarrow} \mathbb{R}^{2}$ with 
$h(\Omega)=\mathbb{D}$ and $K_{h} \in L^{p}(\Omega) \cap L^{q}(B(0, R) \backslash \bar{\Omega})$ for some $1<p, q \leq \infty$, then $\Omega$ is a Sobolev $\left(\frac{2 p}{p-1}, \frac{2 q}{q+1}\right)$-extension domain and $\mathbb{R}^{2} \backslash \bar{\Omega}$ is a Sobolev $\left(\frac{2 q}{q-1}, \frac{2 p}{p+1}\right)$-extension domain.

Inward cuspidal domains with polynomial-type singularity will shows us the sharpness of this result. Also, the combination of this result with the Sobolev extension property of polynomial inward cuspidal domains due to Maz'ya and Poborchi [32-35] will give a new and simpler proof to the necessary part of the main result in [22].

By the result due to Hencl and Koskela in [17], for a homeomorphism of finite distortion $h: \mathbb{R}^{2} \stackrel{\text { onto }}{\longrightarrow} \mathbb{R}^{2}$ with $\exp \left(\lambda K_{h}\right) \in L_{\text {loc }}^{1}\left(\mathbb{R}^{2}\right)$ for some large enough positive constant $\lambda$, we have $K_{h^{-1}} \in L_{\mathrm{loc}}^{p}\left(\mathbb{R}^{2}\right)$ for $1<p<\beta \lambda$ with a constant $\beta<2$ that is independent of $\lambda$. Hence, as an application of Theorem 1.2, we have the following partial answer to the second problem above.

Theorem 1.3 Let $h: \mathbb{R}^{2} \stackrel{\text { onto }}{\longrightarrow} \mathbb{R}^{2}$ be a homeomorphism of finite distortion with $\exp \left(\lambda K_{h}\right) \in$ $L_{\text {loc }}^{1}\left(\mathbb{R}^{2}\right)$ for some $\lambda>0$ large enough. Then $\Omega:=h(\mathbb{D})$ is a Sobolev $\left(\frac{2 p_{\lambda}}{p_{\lambda}-1}, \frac{2 p_{\lambda}}{p_{\lambda}+1}\right)$ extension domain for some $1<p_{\lambda}<\infty$.

However, we have not obtained the best Sobolev extension property for bounded images of the unit disk under global homeomorphisms of finite distortion whose distortions are locally exponentially integrable.

\section{Preliminarily}

The notation $\Omega$ always means a domain in the Euclidean plane $\mathbb{R}^{2} . \widehat{\mathbb{R}^{2}}:=\mathbb{R}^{2} \cup\{\infty\}$ is the one-point compactification of the plane $\mathbb{R}^{2} . B(z, r)$ is a disk with the center $z \in \mathbb{R}^{2}$ and radius $0<r<\infty$. $\mathbb{D}:=B(0,1)$ means the unit disk in $\mathbb{R}^{2}$. For $0<r<R<\infty$, we denote $A(r, R):=B(0, R) \backslash \overline{B(0, r)}$ to be an annulus. Typically, $C$ will be a constant that depends on various parameters and may differ even on the same line of inequalities. For a measurable subset $A \subset \Omega$ with $0<|A|<\infty$ and a function $u \in L_{\mathrm{loc}}^{1}(\Omega), u_{A}$ is the integral average defined by setting

$$
u_{A}:=\int_{A} u(z) d z=\frac{1}{|A|} \int_{A} u(z) d z
$$

Let us give the definition of Sobolev spaces first.

Definition 2.1 Let $1 \leq p \leq \infty$ and $\Omega \subset \mathbb{R}^{2}$ be a domain. we say a function $u \in L_{\text {loc }}^{1}(\Omega)$ belongs to the homogeneous Sobolev space $\dot{W}^{1, p}(\Omega)$ if it is weakly differentiable and its weak derivative satisfies $|\nabla u| \in L^{p}(\Omega)$. The homogeneous Sobolev space $\dot{W}^{1, p}(\Omega)$ is equipped with the semi-norm

$$
\|u\|_{\dot{W}^{1, p}(\Omega)}:=\left(\int_{\Omega}|\nabla u(z)|^{p} d z\right)^{\frac{1}{p}} .
$$

If $u \in L^{p}(\Omega)$ at the same time, we say $u$ is contained in the Sobolev space $W^{1, p}(\Omega)$. The Sobolev space $W^{1, p}(\Omega)$ is equipped with the norm

$$
\|u\|_{W^{1, p}(\Omega)}:=\left(\int_{\Omega}|u(z)|^{p}+|\nabla u(z)|^{p} d z\right)^{\frac{1}{p}} .
$$

Next, we define Sobolev extension domains and homogeneous Sobolev extension domains. 
Definition 2.2 Let $1 \leq q \leq p \leq \infty$. A bounded domain $\Omega \subset \mathbb{R}^{2}$ is said to be a Sobolev $(p, q)$-extension domain, if for every $u \in W^{1, p}(\Omega)$ there exists an extension function $E(u) \in W^{1, q}\left(\mathbb{R}^{2}\right)$ with $\left.E(u)\right|_{\Omega} \equiv u$ and

$$
\|E(u)\|_{W^{1, q}\left(\mathbb{R}^{2}\right)} \leq C\|u\|_{W^{1, p}(\Omega)}
$$

for a constant $C$ that is independent of $u$. Replace $W^{1, p}(\Omega)$ by $\dot{W}^{1, p}(\Omega)$ and replace $W^{1, q}\left(\mathbb{R}^{2}\right)$ by $\dot{W}^{1, q}\left(\mathbb{R}^{2}\right)$ in above, we get the definition of homogeneous Sobolev $(p, q)$ extension domains.

In [19], authors proved that for all $1 \leq p<\infty$ a bounded domain is a homogeneous Sobolev $(p, p)$-extension domain if and only of it is a Sobolev $(p, p)$-extension domain. Their argument also implies that a bounded homogeneous Sobolev $(p, q)$-extension domain is also a Sobolev $(p, q)$-extension domain for $1 \leq q \leq p<\infty$. For the convenience of readers, we present this observation here. We only give the proof on the plane. However, it can be extended to high dimensional Euclidean spaces trivially.

Lemma 2.1 Let $1 \leq q \leq p<\infty$. A bounded homogeneous Sobolev $(p, q)$-extension domain is also a Sobolev $(p, q)$-extension domain.

Proof Let $\Omega \subset \mathbb{R}^{2}$ be a bounded homogeneous Sobolev $(p, q)$-extension domain for $1 \leq q \leq p<\infty$. And let $B \subset \mathbb{R}^{2}$ be a large enough disk with $\Omega \subset \subset B$. Fix $u \in W^{1, p}(\Omega) \subset \dot{W}^{1, p}(\Omega)$. Since $\Omega$ is a homogeneous Sobolev $(p, q)$-extension domain, we have an extension function $E(u) \in \dot{W}^{1, q}\left(\mathbb{R}^{2}\right)$ with $\left.E(u)\right|_{\Omega} \equiv u$ and

$$
\|\nabla E(u)\|_{L^{q}\left(\mathbb{R}^{2}\right)} \leq C\|\nabla u\|_{L^{P}(\Omega)}
$$

for a constant $C$ that is independent of $u$. Since the planar disk $B$ supports $(1, q)$-Poincaré inequality, we have $\left.E(u)\right|_{B} \in W^{1, q}(B)$. Then, as claimed in [19, Lemma 4.1], by combining results in $[20$, Section 2.3$]$ and $[14,(7.45)]$, we obtain

$$
\begin{aligned}
& \left(\int_{B}\left|E(u)(z)-u_{\Omega}\right|^{q} d z\right)^{\frac{1}{q}} \\
& \quad \leq 2^{n} \operatorname{diam}(B)\left(\frac{|B|}{|\Omega|}\right)^{\frac{1}{q}}\left(\int_{B}|\nabla E(u)(z)|^{q} d z\right)^{\frac{1}{q}} \\
& \quad \leq C\left(\int_{\Omega}|\nabla u(z)|^{p} d z\right)^{\frac{1}{p}} .
\end{aligned}
$$

By (2.1), the triangle inequality and the Hölder inequality, we obtain the desired inequality that

$$
\begin{aligned}
& \left(\int_{B}|E(u)(z)|^{q}+|\nabla E(u)(z)|^{q} d z\right)^{\frac{1}{q}} \\
& \quad \leq C\left(\int_{\Omega}|\nabla u(z)|^{p}\right)^{\frac{1}{p}}+C\left(\frac{|B|}{|\Omega|}\right)^{\frac{1}{q}}\left(\int_{\Omega}|u(z)|^{q} d z\right)^{\frac{1}{q}} \\
& \quad \leq C\left(\int_{\Omega}|u(z)|^{p} d z+|\nabla u(z)|^{p} d z\right)^{\frac{1}{p}} .
\end{aligned}
$$


Let us define homeomorphisms of finite distortion and $L^{p}$-quasidisks.

Definition 2.3 We say a homeomorphism $h: \Omega \stackrel{\text { onto }}{\longrightarrow} h(\Omega)$ has finite distortion if $h \in$ $W_{\text {loc }}^{1,1}\left(\Omega, \mathbb{R}^{2}\right)$ and there is a measurable function $K: \Omega \rightarrow[1, \infty]$ with $K(z)<\infty$ almost everywhere such that

$$
|D h(z)|^{2} \leq K(z) J_{h}(z) \text { for almost all } z \in \Omega .
$$

For a homeomorphism of finite distortion, we define the optimal distortion function by setting

$$
K_{h}(z):= \begin{cases}\frac{|D h(z)|^{2}}{J_{h}(z)}, & \text { for } z \in\left\{J_{h}>0\right\}, \\ 1, & \text { for } z \in\left\{J_{h}=0\right\} .\end{cases}
$$

Definition 2.4 Let $1 \leq p \leq \infty$. A bounded simply connected domain $\Omega \subset \mathbb{R}^{2}$ is said to be a $L^{p}$-quasidisk, if there exists a global homeomorphism of finite distortion $h: \mathbb{R}^{2} \stackrel{\text { onto }}{\longrightarrow} \mathbb{R}^{2}$ with $h(\Omega)=\mathbb{D}$ and $K_{h} \in L_{\text {loc }}^{p}\left(\mathbb{R}^{2}\right)$.

Let $\Omega \subset \mathbb{R}^{2}$ be a bounded simply connected domain. A self homeomorphism $h: \mathbb{R}^{2} \stackrel{\text { onto }}{\longrightarrow}$ $\mathbb{R}^{2}$ is called a reflection over the boundary $\partial \Omega$ if $h(\Omega)=\widehat{\mathbb{R}^{2}} \backslash \bar{\Omega}$ and $h(z)=z$ for every $z \in \partial \Omega$. Based on the homeomorphism of finite distortion in Theorem 1.2, we can construct a reflection $\mathcal{R}_{h}: \mathbb{R}^{2} \stackrel{\text { onto }}{\longrightarrow} \mathbb{R}^{2}$ over $\partial \Omega$ which can induce a bounded linear extension operator from $\dot{W}^{1, \frac{2 p}{p+1}}(\Omega)$ to $\dot{W}^{1, \frac{2 q}{q-1}}\left(\mathbb{R}^{2}\right)$ and a bounded linear extension operator from $\dot{W}^{1, \frac{2 q}{q+1}}\left(\mathbb{R}^{2} \backslash\right.$ $\bar{\Omega})$ to $\dot{W}^{1, \frac{2 p}{p-1}}\left(\mathbb{R}^{2}\right)$. We say a reflection $\mathcal{R}: \widehat{\mathbb{R}^{2}} \stackrel{\text { onto }}{\longrightarrow} \widehat{\mathbb{R}^{2}}$ over $\partial \Omega$ induces a bounded linear Sobolev $(p, q)$-extension operator for $\Omega$ with $1 \leq q \leq p<\infty$, if there exists a bounded Lipschitz domain $U$ containing $\partial \Omega$ such that, for every function $u \in \dot{W}^{1, p}(\Omega)$, the function $v$ defined by setting $v=u$ on $U \cap \Omega$ and $v=u \circ \mathcal{R}$ on $U \backslash \bar{\Omega}$ has a representative which belongs to the Sobolev space $\dot{W}^{1, q}(U)$ and we have

$$
\|v\|_{\dot{W}^{1, q}(U)} \leq C\|u\|_{\dot{W}^{1, p}(\Omega)}
$$

with a positive constant $C$ that is independent of $u$. Similarly, we say the reflection $\mathcal{R}$ induces a bounded linear Sobolev $(p, q)$-extension operator for $\mathbb{R}^{2} \backslash \bar{\Omega}$ with $1 \leq q \leq p<$ $\infty$, if for every $u \in \dot{W}^{1, p}\left(\mathbb{R}^{2} \backslash \bar{\Omega}\right)$, the function $\tilde{v}$ defined by setting $\tilde{v}=u$ on $U \backslash \bar{\Omega}$ and $\tilde{v}=u \circ \mathcal{R}$ on $U \cap \Omega$ has a representative which belongs to the Sobolev space $\dot{W}^{1, q}(U)$ and we have

$$
\|\tilde{v}\|_{\dot{W}^{1, q}(U)} \leq C\|u\|_{\dot{W}^{1, p}\left(\mathbb{R}^{2} \backslash \bar{\Omega}\right)}
$$

with a positive constant $C$ that is independent of $u$. By using a suitable cut-off function, it is easy to see $\Omega$ or $\mathbb{R}^{2} \backslash \bar{\Omega}$ is a homogeneous Sobolev $(p, q)$-extension domain. Here the introduction of the bounded open set $U$ is a convenient way to overcome the non-essential difficulty that functions in $\dot{W}^{1, p}(G)$ do not necessarily belong to $\dot{W}^{1, q}(G)$ when $1 \leq q<$ $p<\infty$ and $G$ has infinite volume. The following technical lemma justifies our terminology.

Proposition 2.1 Let $\Omega \subset \mathbb{R}^{2}$ be a bounded domain with $|\partial \Omega|=0$ and $\mathcal{R}: \widehat{\mathbb{R}^{2}} \rightarrow \widehat{\mathbb{R}^{2}}$ be a reflection over $\partial \Omega$. If $\mathcal{R}$ induces a bounded linear extension operator from $\dot{W}^{1, p}(\Omega)$ to $\dot{W}^{1, q}\left(\mathbb{R}^{2}\right)$ in the sense of $(2.4)$ (from $\dot{W}^{1, p}\left(\mathbb{R}^{2} \backslash \bar{\Omega}\right)$ to $\dot{W}^{1, q}\left(\mathbb{R}^{2}\right)$, respectively) for some $1<q \leq p<\infty$, then $\Omega\left(\mathbb{R}^{2} \backslash \bar{\Omega}\right.$, respectively) is a homogeneous Sobolev $(p, q)$-extension domain with a linear extension operator. 
Proof We only consider the case of $\Omega$, since the case of $\mathbb{R}^{2} \backslash \bar{\Omega}$ is analogous. Let $U \subset \mathbb{R}^{2}$ be the corresponding Lipschitz domain which contains $\partial \Omega$. For a given function $u \in \dot{W}^{1, p}(\Omega)$, we define a function $E_{\mathcal{R}}(u)$ by setting

$$
E_{\mathcal{R}}(u)(z):= \begin{cases}u(\mathcal{R}(z)), & \text { for } z \in U \backslash \bar{\Omega}, \\ 0, & \text { for } z \in \partial \Omega, \\ u(z), & \text { for } z \in \Omega .\end{cases}
$$

Then $E_{\mathcal{R}}(u)$ has a representative that belongs to $\dot{W}^{1, q}(U)$ with

$$
\left\|E_{\mathcal{R}}(u)\right\|_{\dot{W}^{1, q}(U)} \leq C\|u\|_{\dot{W}^{1, p}(\Omega)} .
$$

Since $U \subset \mathbb{R}^{2}$ is a bounded Lipschitz domain, by the result due to Jones [24], for every $1<q<\infty$, there exists a bounded linear extension operator $L: \dot{W}^{1, q}(U) \rightarrow \dot{W}^{1, q}\left(\mathbb{R}^{2}\right)$. For every function $u \in \dot{W}^{1, p}(\Omega)$, we define an extension function $\tilde{E}_{\mathcal{R}}(u) \in \dot{W}^{1, q}\left(\mathbb{R}^{2}\right)$ by setting

$$
\tilde{E}_{\mathcal{R}}(u):=L\left(E_{\mathcal{R}}(u)\right) .
$$

Then, we have $\left.\tilde{E}_{\mathcal{R}}(u)\right|_{\Omega} \equiv u$ with

$$
\left\|\tilde{E}_{\mathcal{R}}(u)\right\|_{\dot{W}^{1, q}\left(\mathbb{R}^{2}\right)} \leq C\|u\|_{\dot{W}^{1, p}(\Omega)}
$$

for a constant $C$ independent of $u$.

A strictly increasing function $\rho:[0, \infty) \stackrel{\text { onto }}{\longrightarrow}[0, \infty)$ of class $C^{1}(0, \infty) \cap C[0, \infty)$ is called a cuspidal function if $\rho(1)=1, \rho^{\prime}$ is increasing in $(0, \infty)$ and

$$
\lim _{x \rightarrow 0^{+}} \rho^{\prime}(x)=0 \text {. }
$$

The corresponding inward and outward cuspidal domains are defined by setting

$$
\Omega_{\rho}^{i}:=B(0,1) \backslash\{(x, y) \in[0,1] \times \mathbb{R}:|y|<\rho(x)\}
$$

and

$$
\Omega_{\rho}^{o}:=B((2,0), \sqrt{2}) \cup\{(x, y) \in(0,1] \times \mathbb{R}:|y|<\rho(x)\}
$$

respectively. See the Figure below for the exemplary cuspidal function $\rho(x)=x^{\frac{4}{3}}$ Fig. 1 .

If the cuspidal function is $\rho(x)=x^{s}$ for some $1<s<\infty$, the corresponding inward and outward cuspidal domains are called polynomial inward and outward cuspidal domains

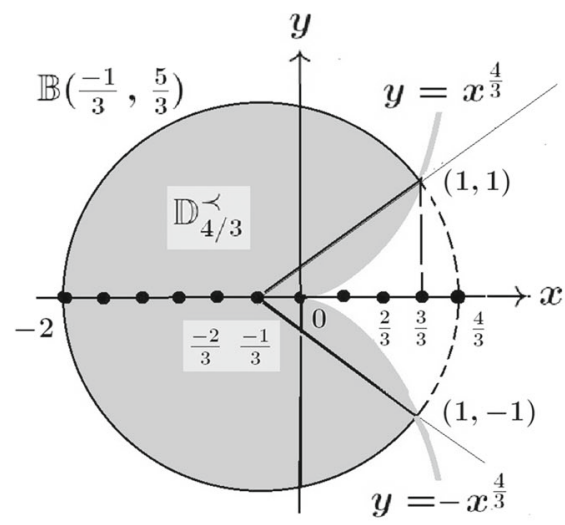

Fig. 1 Inward and outward cuspidal domains

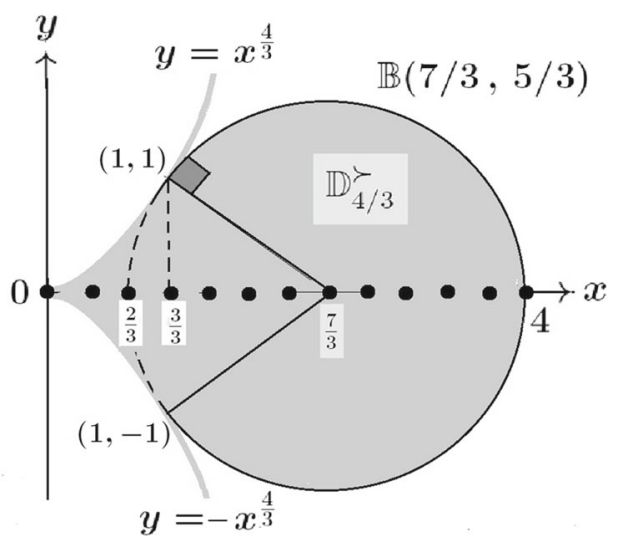


with the degree $s$. The following result about Sobolev extension property of polynomial inward and outward cuspidal domains is doe to Maz'ya and Poborchi [32-35].

Proposition 2.2 The polynomial outward cuspidal domain $\Omega_{t^{s}}^{o}$ is a Sobolev $(p, q)$ extension domain for $1 \leq q<p<\infty$ if and only if $s<\frac{2 p}{q}-1$. The polynomial inward cuspidal domain $\Omega_{t^{s}}^{i}$ is a Sobolev $(p, q)$-extension domain for $1<q<p \leq \infty$ if and only if $s<\frac{p q+p-2 q}{p q-p}$. And for every $1<s<\infty$, the polynomial inward cuspidal domain $\Omega_{t^{s}}^{i}$ is a Sobolev $(1,1)$-extension domain.

By combining Theorem 1.2 and Proposition 2.2, we can give a new and simpler proof to the necessary part of the following proposition which is the main result in [22]. The proof of the sufficient part comes from the construction of desired homeomorphisms of finite distortion in [22].

Proposition 2.3 Let $\Omega_{t^{s}}^{i} \subset \mathbb{R}^{2}$ be a polynomial inward cuspidal domain with the degree $1<s<\infty$. Then there exists a homeomorphism of finite distortion $h: \mathbb{R}^{2} \stackrel{\text { onto }}{\longrightarrow} \mathbb{R}^{2}$ with $h\left(\Omega_{t^{s}}^{i}\right)=\mathbb{D}$ and $K_{h} \in L^{p}\left(\Omega_{t^{s}}^{i}\right) \cap L^{q}\left(B(0,2) \backslash \overline{\Omega_{t^{s}}^{i}}\right)$ for $1<p, q \leq \infty$ with $\min \{p, q\}<\infty$ if and only if $s<\frac{p q+p+2 q}{p q-p}$.

Combining Propositions 2.2 and 2.3, we can also obtain the sharpness of Theorem 1.2. The polynomial inward cuspidal domain $\Omega_{t^{s}}^{i}$ shows that there exists a bounded simply connected domain $\Omega \subset \mathbb{R}^{2}$ which satisfies assumptions of Theorem 1.2, such that we can not hope that we can obtain a better Sobolev extension property than $\Omega$ is a Sobolev $\left(\frac{2 p}{p-1}, \frac{2 q}{q+1}\right)$-extension domain and $\mathbb{R}^{2} \backslash \bar{\Omega}$ is a Sobolev $\left(\frac{2 q}{q-1}, \frac{2 p}{p+1}\right)$-extension domain.

\section{Proofs of Theorems 1.1 and 1.2}

\subsection{Proof of Theorem 1.1}

By [22, Theorem 1.8], every simply connected Jordan domain with a rectifiable boundary is a $L^{1}$-quasidisk. Hence, an outward cuspidal domain $\Omega_{\rho}^{o}$ with cuspidal function $\rho(x)=$ $e / \exp \left(\frac{1}{x}\right)$ is a $L^{1}$-quasidisk. By Proposition 2.2, $\Omega_{\rho}^{o}$ cannot be a Sobolev $(p, q)$-extension domain for any $1 \leq q \leq p<\infty$.

\subsection{Proof of Theorem 1.2}

Let $\Omega \subset \mathbb{R}^{2}$ be a bounded simply connected domain with $\bar{\Omega} \subset \subset B(0, R)$ for a large enough $R>0$. Suppose there exists a homeomorphism of finite distortion $h: \mathbb{R}^{2} \stackrel{\text { onto }}{\longrightarrow} \mathbb{R}^{2}$ with $h(\Omega)=\mathbb{D}$ and

$$
\int_{\Omega} K_{h}^{p}(z) d z+\int_{B(0, R) \backslash \bar{\Omega}} K_{h}^{q}(z) d z<\infty
$$

The circle inversion map $\mathcal{R}: \widehat{\mathbb{R}^{2}} \stackrel{\text { onto }}{\longrightarrow} \widehat{\mathbb{R}^{2}}$,

$$
\mathcal{R}(z):= \begin{cases}\frac{z}{|z|^{2}}, & \text { if } z \neq 0, \\ \infty, & \text { if } z=0 .\end{cases}
$$


is an anticonformal reflection over the unit circle $\partial \mathbb{D}$, which means that at every point it preserves angles and reverses orientation. Then a self-homeomorphism $\widetilde{\mathcal{R}}: \widehat{\mathbb{R}^{2}} \stackrel{\text { onto }}{\longrightarrow} \widehat{\mathbb{R}^{2}}$ defined on every $z \in \widehat{\mathbb{R}^{2}}$ by setting

$$
\widetilde{\mathcal{R}}(z):=h^{-1} \circ \mathcal{R} \circ h(z)
$$

is a reflection over the boundary $\partial \Omega$.

With respect to different domains $\Omega$ and $\mathbb{R}^{2} \backslash \bar{\Omega}$, we divide the full proof of Theorem 1.2 into two parts. First, let us prove the Sobolev extension property of the bounded domain $\Omega$.

Theorem 3.1 Under the assumption of Theorem 1.2, $\Omega$ is a Sobolev $\left(\frac{2 p}{p-1}, \frac{2 q}{q+1}\right)$-extension domain.

Proof By Lemma 2.1, it is sufficient to show that $\Omega$ is a homogeneous Sobolev $\left(\frac{2 p}{p-1}, \frac{2 q}{q+1}\right)$-extension domain. Let $u \in \dot{W}^{1, \frac{2 p}{p-1}}(\Omega)$ be arbitrary. We define an extension function $E_{\widetilde{\mathcal{R}}}(u)$ on $B(0, R)$ by setting

$$
E_{\widetilde{\mathcal{R}}}(u)(z):= \begin{cases}u(\widetilde{\mathcal{R}}(z)), & \text { if } z \in B(0, R) \backslash \bar{\Omega}, \\ 0, & \text { if } z \in \partial \Omega, \\ u(z), & \text { if } z \in \Omega .\end{cases}
$$

By Proposition 2.1, it suffices to prove that $E_{\widetilde{\mathcal{R}}}(u) \in \dot{W}^{1, \frac{2 q}{q+1}}(B(0, R))$ with the following inequality that

$$
\left(\int_{B(0, R)}\left|D E_{\widetilde{\mathcal{R}}}(u)(z)\right|^{\frac{2 q}{q+1}} d z\right)^{\frac{q+1}{2 q}} \leq C\left(\int_{\Omega}|D u(z)|^{\frac{2 p}{p-1}} d z\right)^{\frac{p-1}{2 p}}
$$

for a positive constant $C$ independent of $u$. Since $1<\frac{2 q}{q+1} \leq 2 \leq \frac{2 p}{p-1}<\infty$, the Hölder inequality implies

$$
\left(\int_{\Omega}\left|D E_{\widetilde{\mathcal{R}}}(u)(z)\right|^{\frac{2 q}{q+1}} d z\right)^{\frac{q+1}{2 q}} \leq C\left(\int_{\Omega}|D u(z)|^{\frac{2 p}{p-1}} d z\right)^{\frac{p-1}{2 p}} .
$$

By [18, Theorem 4.13], $\partial \Omega$ must be of measure zero. Hence, we only need to prove

$$
\left(\int_{B(0, R) \backslash \bar{\Omega}}\left|D E_{\widetilde{\mathcal{R}}}(u)(z)\right|^{\frac{2 q}{q+1}} d z\right)^{\frac{q+1}{2 q}} \leq C\left(\int_{\Omega}|D u(z)|^{\frac{2 p}{p-1}} d z\right)^{\frac{p-1}{2 p}} .
$$

We define a function $v$ on $\mathbb{D}$ by setting $v(z):=u \circ h^{-1}(z)$ for every $z \in \mathbb{D}$ and define an extension function $E_{\mathcal{R}}(v)$ on $h(B(0, R))$ with $z=(x, y)$ by setting

$$
E_{\mathcal{R}}(v)(z):= \begin{cases}v(\mathcal{R}(z)), & \text { if } z \in h(B(0, R)) \backslash \overline{\mathbb{D}}, \\ 0, & \text { if } z \in \partial \mathbb{D}, \\ v(z), & \text { if } z \in \mathbb{D} .\end{cases}
$$

By the definitions of functions and reflections, for every $z \in B(0, R)$, we have

$$
E_{\tilde{\mathcal{R}}}(u)(z)=E_{\mathcal{R}}(v)(h(z)) .
$$

We divide the remaining argument into three steps. 
Step 1: We would like to show $v \in \dot{W}^{1,2}(\mathbb{D})$ with

$$
\left(\int_{\mathbb{D}}|D v(z)|^{2} d z\right)^{\frac{1}{2}} \leq C\left(\int_{\Omega}|D u(z)|^{\frac{2 p}{p-1}} d z\right)^{\frac{p-1}{2 p}}
$$

for a positive constant $C$ that is independent of $u$. By [18, Theorem 1.6 and Theorem 2.24], $h^{-1} \in W^{1,1}\left(\mathbb{D}, \mathbb{R}^{2}\right)$ and it is differentiable almost everywhere on $\mathbb{D}$. Hence, there exists a measurable subset $G \subset \mathbb{D}$ with $|G|=|\mathbb{D}|$ and $h^{-1}$ is differentiable on every point $z \in G$. By the chain rule, for every $z \in G$, we have

$$
|D v(z)| \leq\left|D u\left(h^{-1}(z)\right)\right| \cdot\left|D h^{-1}(z)\right| .
$$

Set $A \subset G$ to be the subset such that $J_{h^{-1}}(z)>0$ for every $z \in A$. Then by [18, Theorem 1.6], $\left|D h^{-1}(z)\right|=0$ for almost every $z \in G \backslash A$. Hence, we have

$$
\begin{aligned}
\int_{\mathbb{D}}|D v(z)|^{2} d z & =\int_{G}|D v(z)|^{2} d z \\
& \leq \int_{A}\left|D u\left(h^{-1}(z)\right)\right|^{2} \cdot\left|D h^{-1}(z)\right|^{2} d z .
\end{aligned}
$$

If $p=\infty$, then $\left.h\right|_{\Omega}$ is quasiconformal. By the fact that the inverse of a quasiconformal mapping is also quasiconformal, we have

$$
\begin{aligned}
& \int_{A}\left|D u\left(h^{-1}(z)\right)\right|^{2} \cdot\left|D h^{-1}(z)\right|^{2} d z \\
= & \int_{A}\left|D u\left(h^{-1}(z)\right)\right|^{2} J_{h^{-1}}(z) \frac{\left|D h^{-1}(z)\right|^{2}}{J_{h^{-1}}(z)} d z \\
\leq & C \int_{A}\left|D u\left(h^{-1}(z)\right)\right|^{2} J_{h^{-1}}(z) d z .
\end{aligned}
$$

If $1<p<\infty$, the Hölder inequality implies

$$
\begin{aligned}
& \int_{A}\left|D u\left(h^{-1}(z)\right)\right|^{2} \cdot\left|D h^{-1}(z)\right|^{2} d z \\
= & \int_{A}\left|D u\left(h^{-1}(z)\right)\right|^{2} J_{h^{-1}}^{\frac{p-1}{p}}(z) \frac{\left|D h^{-1}(z)\right|^{2}}{J_{h^{-1}}^{\frac{p-1}{p}}(z)} d z \\
\leq & \left(\int_{A}\left|D u\left(h^{-1}(z)\right)\right|^{\frac{2 p}{p-1}} J_{h^{-1}}(z) d z\right)^{\frac{p-1}{p}} \\
& \times\left(\int_{A} \frac{\left|D h^{-1}(z)\right|^{2 p}}{J_{h^{-1}}^{p}(z)} J_{h^{-1}}(z) d z\right)^{\frac{1}{p}} .
\end{aligned}
$$

For every $1<p \leq \infty$, the change of variables formula implies

$$
\int_{A}\left|D u\left(h^{-1}(z)\right)\right|^{\frac{2 p}{p-1}} J_{h^{-1}}(z) d z \leq \int_{h^{-1}(A)}|D u(z)|^{\frac{2 p}{p-1}} d z .
$$

By [18, Lemma A.29], $h$ is differentiable on every $w \in h^{-1}(A)$ with $D h(w)=$ $\left(D h^{-1}(z)\right)^{-1}$ for $w=h^{-1}(z)$. Hence, for every $z \in A$ with $w=h^{-1}(z)$, we have

$$
K_{h^{-1}}(z)=\frac{\left|D h^{-1}(z)\right|^{2}}{J_{h^{-1}}(z)}=\frac{|D h(w)|^{2}}{J_{h}(w)}=K_{h}(w) .
$$


Hence, the change of variables formula implies

$$
\int_{A} \frac{\left|D h^{-1}(z)\right|^{2 p}}{J_{h^{-1}}^{p}(z)} J_{h^{-1}}(z) d z \leq \int_{\Omega} K_{h}^{p}(z) d z .
$$

By combing inequalities (3.8), (3.9), (3.10), (3.11) and (3.12), we obtain the desired inequality (3.6).

Step 2: We prove $E_{\mathcal{R}}(v) \in \dot{W}^{1,2}(h(B(0, R)))$ with

$$
\int_{h(B(0, R))}\left|D E_{\mathcal{R}}(v)(z)\right|^{2} d z \leq C \int_{\mathbb{D}}|D v(z)|^{2} d z
$$

for a positive constant $C$ that is independent of $v$. By Lemma 2.1, $v \in \dot{W}^{1,2}(\mathbb{D})=W^{1,2}(\mathbb{D})$. Since $C^{\infty}\left(\mathbb{R}^{2}\right) \cap W^{1,2}(\mathbb{D})$ is dense in $W^{1,2}(\mathbb{D})$, we can find a sequence of functions $v_{k} \in$ $C^{\infty}\left(\mathbb{R}^{2}\right) \cap W^{1,2}(\mathbb{D})$ with

$$
\lim _{k \rightarrow \infty} v_{k}(z)=v(z)
$$

for almost every $z \in \mathbb{D}$, and

$$
\lim _{k \rightarrow \infty}\left\|v_{k}-v\right\|_{\dot{W}^{1,2}(\mathbb{D})}=0 .
$$

By passing to a subsequence if necessary, we can also assume the sequence of weak gradients $\left\{D v_{k}\right\}$ converges to $D v$ almost everywhere on $\mathbb{D}$. For every $v_{k} \in C^{\infty}\left(\mathbb{R}^{2}\right) \cap W^{1,2}(\mathbb{D})$, we define the extension function $E_{\mathcal{R}}\left(v_{k}\right)$ as in Eq. 3.5. Since $v_{k} \in C^{\infty}\left(\mathbb{R}^{2}\right) \cap W^{1,2}(\mathbb{D})$, by resetting the definition of $E_{\mathcal{R}}\left(v_{k}\right)$ on the measure-zero set $\partial \mathbb{D}$, we can show that $E_{\mathcal{R}}\left(v_{k}\right)$ is $A C L$ on $h(B(0, R))$. By definition (3.5) and the fact that $\mathcal{R}$ is anticonformal, it is easy to see that we have

$$
\int_{h(B(0, R))}\left|D E_{\mathcal{R}}\left(v_{k}\right)(z)\right|^{2} d z \leq C \int_{\mathbb{D}}\left|D v_{k}(z)\right|^{2} d z,
$$

for a positive constant $C$ independent of $k$. Since $v_{k}$ converges to $v$ almost everywhere on $\mathbb{D}$, by (3.5), $E_{\mathcal{R}}\left(v_{k}\right)$ converges to $E_{\mathcal{R}}(v)$ almost everywhere on $h(B(0, R))$. Since $\left\{D v_{k}\right\}$ converges to $D v$ almost everywhere on $\mathbb{D}$, by the definitions of $E_{\mathcal{R}}\left(v_{k}\right)$ and $E_{\mathcal{R}}(v)$ in Eq. 3.5, $\left\{D E_{\mathcal{R}}\left(v_{k}\right)\right\}$ converges to $D E_{\mathcal{R}}(v)$ almost everywhere on $h(B(0, R))$. By Eqs. 3.14 and 3.15 , we have

$$
\lim _{k \rightarrow \infty} \int_{h(B(0, R))}\left|D E_{\mathcal{R}}\left(v_{k}\right)(z)-D E_{\mathcal{R}}(v)(z)\right|^{2} d z=0 .
$$

Then by Theorem 1 in [31, Section 1.1.13], $E_{\mathcal{R}}(v) \in \dot{W}^{1,2}(h(B(0, R)))$ with the weak gradient $D E_{\mathcal{R}}(v)$. Combine (3.14), (3.15) and (3.16), we obtain

$$
\int_{h(B(0, R))}\left|D E_{\mathcal{R}}(v)(z)\right|^{2} d z \leq C \int_{\mathbb{D}}|D v(z)|^{2} d z,
$$

with $C$ a positive constant that is independent of $u$.

Step 3: We prove $E_{\widetilde{\mathcal{R}}}(u) \in \dot{W}^{1, \frac{2 q}{q+1}}(B(0, R))$ and we have

$$
\begin{aligned}
& \left(\int_{B(0, R) \backslash \bar{\Omega}}\left|D E_{\widetilde{\mathcal{R}}}(u)(z)\right|^{\frac{2 q}{q+1}} d z\right)^{\frac{q+1}{2 q}} \\
\leq & C\left(\int_{h(B(0, R))}\left|D E_{\mathcal{R}}(v)(z)\right|^{2} d z\right)^{\frac{1}{2}}
\end{aligned}
$$

for a positive constant $C$ that is independent of $u$. As we know, $E_{\widetilde{\mathcal{R}}}(u)(z)=E_{\mathcal{R}}(v)(h(z))$ for every $z \in B(0, R)$. By [18, Theorem 1.7 and Theorem 2.24], $h$ is differentiable almost 
everywhere on $B(0, R) \backslash \bar{\Omega}$ with positive determinant value. Hence, there exists a measurable subset $\widehat{G} \subset B(0, R) \backslash \bar{\Omega}$ with $|\widehat{G}|=|B(0, R) \backslash \bar{\Omega}|$ and $h$ is differentiable on every point in $z \in \widehat{G}$ with $J_{h}(z)>0$. By the chain rule, for every $z \in \widehat{G}$, we have

$$
\left|D E_{\widetilde{\mathcal{R}}}(u)(z)\right| \leq\left|D E_{\mathcal{R}}(v(h(z)))\right| \cdot|D h(z)| .
$$

If $q=\infty$, then $h$ restricts to $B(0, R) \backslash \bar{\Omega}$ is quasiconformal. Then we have

$$
\begin{aligned}
\int_{B(0, R) \backslash \bar{\Omega}}\left|D E_{\widetilde{\mathcal{R}}}(u)(z)\right|^{2} d z & \\
& \leq \int_{B(0, R) \backslash \bar{\Omega}}\left|D E_{\mathcal{R}}(v)(h(z))\right|^{2} J_{h}(z) \cdot \frac{|D h(z)|^{2}}{J_{h}(z)} d z \\
& \leq C \int_{B(0, R) \backslash \bar{\Omega}}\left|D E_{\mathcal{R}}(v)(h(z))\right|^{2} J_{h}(z) d z .
\end{aligned}
$$

If $1<q<\infty$, then we have

$$
\begin{gathered}
\int_{B(0, R) \backslash \bar{\Omega}}\left|D E_{\widetilde{\mathcal{R}}}(u)(z)\right|^{\frac{2 q}{q+1}} d z=\int_{\widehat{G}}\left|D E_{\widetilde{\mathcal{R}}}(u)(z)\right|^{\frac{2 q}{q+1}} d z \\
\leq \int_{\widehat{G}}\left|D E_{\mathcal{R}}(v)(h(z))\right|^{\frac{2 q}{q+1}} \cdot|D h(z)|^{\frac{2 q}{q+1}} d z .
\end{gathered}
$$

The Hölder inequality implies

$$
\begin{aligned}
& \int_{\widehat{G}}\left|D E_{\mathcal{R}}(v)(h(z))\right|^{\frac{2 q}{q+1}} \cdot|D h(z)|^{\frac{2 q}{q+1}} d z \\
\leq & \int_{\widehat{G}}\left|D E_{\mathcal{R}}(v)(h(z))\right|^{\frac{2 q}{q+1}} J_{h}^{\frac{q}{q+1}}(z) \frac{|D h(z)|^{\frac{2 q}{q+1}}}{J_{h}^{\frac{q}{q+1}}(z)} d z \\
\leq & \left(\int_{\widehat{G}}\left|D E_{\mathcal{R}}(v)(h(z))\right|^{2} J_{h}(z) d z\right)^{\frac{q}{q+1}} \\
& \times\left(\int_{\widehat{G}}\left(\frac{|D h(z)|^{2}}{J_{h}(z)}\right)^{q} d z\right)^{\frac{1}{q}} .
\end{aligned}
$$

The change of variables formula implies

$$
\int_{\widehat{G}}\left|D E_{\mathcal{R}}(v)(h(z))\right|^{2} J_{h}(z) d z \leq \int_{h(B(0, R))}\left|D E_{\mathcal{R}}(v)(z)\right|^{2} d z
$$

By the definition of distortion function and the integral condition (3.1), we have

$$
\int_{\widehat{G}}\left(\frac{|D h(z)|^{2}}{J_{h}(z)}\right)^{q} d z \leq \int_{B(0, R) \backslash \bar{\Omega}} K_{h}^{q}(z) d z<\infty .
$$

Combine inequalities (3.20)-(3.24), we obtain the desired inequality (3.18).

Finally, combine inequalities (3.6), (3.13) and (3.18), we obtain the desired inequality (3.4) and finish the proof of Theorem 1.2.

Next, we consider the Sobolev extension property of the complementary domain $\mathbb{R}^{2} \backslash \bar{\Omega}$. 
Theorem 3.2 Under the assumption of Theorem 1.2, $\mathbb{R}^{2} \backslash \bar{\Omega}$ is a Sobolev $\left(\frac{2 q}{q-1}, \frac{2 p}{p+1}\right)$ extension domain.

The idea of the proof is very similar with the proof of last theorem. The main difference is that $\mathbb{R}^{2} \backslash \bar{\Omega}$ is unbounded here. Hence, we give a sketch proof here.

Proof From the geometrical viewpoint, it is easy to see $\mathbb{R}^{2} \backslash \bar{\Omega}$ has same Sobolev extension property with the bounded domain $B(0, R) \backslash \bar{\Omega}$. Hence, by Lemma 2.1 , it suffices to prove $B(0, R) \backslash \bar{\Omega}$ is a homogeneous Sobolev $\left(\frac{2 q}{q-1}, \frac{2 p}{p+1}\right)$-extension domain. Since $h: \mathbb{R}^{2} \stackrel{\text { onto }}{\longrightarrow} \mathbb{R}^{2}$ is a homeomorphism with $h(\Omega)=\mathbb{D}$, there exist two positive constants $\epsilon_{1}>0$ and $\epsilon_{2}>0$ such that $B\left(0,1+\epsilon_{1}\right) \subset h(B(0, R))$ and

$$
\mathcal{R}\left(A\left(1-\epsilon_{2}, 1+\epsilon_{1}\right)\right)=A\left(1-\epsilon_{2}, 1+\epsilon_{1}\right) .
$$

We define $\mathcal{A}:=h^{-1}\left(A\left(1-\epsilon_{2}, 1\right)\right), \mathbf{U}:=(B(0, R) \backslash \Omega) \cup \mathcal{A}$ and $\widetilde{\mathbf{U}}:=h(\mathbf{U})$. Let $u \in$ $\dot{W}^{1, \frac{2 q}{q-1}}\left(\mathbb{R}^{2} \backslash \bar{\Omega}\right)$ be arbitrary. Then

$$
\left.u\right|_{B(0, R) \backslash \bar{\Omega}} \in \dot{W}^{1, \frac{2 q}{q-1}}(B(0, R) \backslash \bar{\Omega}) .
$$

To simplify the notation, we still denote $\left.u\right|_{B(0, R) \backslash \bar{\Omega}}$ by $u$. Then we define a function $E_{\widetilde{\mathcal{R}}}(u)$ on $\mathbf{U}$ by setting

$$
E_{\widetilde{\mathcal{R}}}(u)(z):= \begin{cases}u(\widetilde{\mathcal{R}}(z)), & \text { if } z \in \mathcal{A}, \\ 0, & \text { if } z \in \partial \Omega, \\ u(z), & \text { if } z \in B(0, R) \backslash \bar{\Omega} .\end{cases}
$$

It suffices to prove $E_{\widetilde{\mathcal{R}}}(u) \in \dot{W}^{1, \frac{2 p}{p+1}}(\mathbf{U})$ with

$$
\begin{aligned}
& \left(\int_{\mathbf{U}}\left|D E_{\widetilde{\mathcal{R}}}(u)(z)\right|^{\frac{2 p}{p+1}} d z\right)^{\frac{p+1}{2 p}} \\
\leq & C\left(\int_{B(0, R) \backslash \bar{\Omega}}|D u(z)|^{\frac{2 q}{q-1}} d z\right)^{\frac{q-1}{2 q}} .
\end{aligned}
$$

for a positive constant $C$ that is independent of $u$. By the definition of $E_{\widetilde{\mathcal{R}}}(u)$, the Hölder inequality implies

$$
\begin{aligned}
& \left(\int_{B(0, R) \backslash \bar{\Omega}}\left|D E_{\widetilde{\mathcal{R}}}(u)(z)\right|^{\frac{2 p}{p+1}}\right)^{\frac{p+1}{2 p}} \\
\leq & C\left(\int_{B(0, R) \backslash \bar{\Omega}}|D u(z)|^{\frac{2 q}{q-1}} d z\right)^{\frac{q-1}{2 q}}
\end{aligned}
$$

for a positive constant $C$ that is independent of $u$. By [18, Theorem 4.13], $\partial \Omega$ must be of measure zero. Hence, it suffices to prove the inequality

$$
\begin{aligned}
& \left(\int_{\mathcal{A}}\left|D E_{\widetilde{\mathcal{R}}}(u)(z)\right|^{\frac{2 p}{p+1}} d z\right)^{\frac{p+1}{2 p}} \\
\leq & C\left(\int_{B(0, R) \backslash \bar{\Omega}}|D u(z)|^{\frac{2 q}{q-1}} d z\right)^{\frac{q-1}{2 q}}
\end{aligned}
$$


for a positive constant $C$ that is independent of $u$. We define a function $v \in h(B(0, R) \backslash \bar{\Omega})$ by setting $v(z):=u\left(h^{-1}(z)\right)$ for every $z \in h(B(0, R) \backslash \bar{\Omega})$. By a similar argument to the inequality (3.6), we obtain $v \in \dot{W}^{1,2}(h(B(0, R) \backslash \bar{\Omega}))$ with

$$
\left(\int_{h(B(0, R) \backslash \bar{\Omega})}|D v(z)|^{2} d z\right)^{\frac{1}{2}} \leqq C\left(\int_{B(0, R) \backslash \bar{\Omega}}|D u(z)|^{\frac{2 q}{q-1}}\right)^{\frac{q-1}{2 q}}
$$

for a positive constant $C$ that is independent of $u$. Define an extension function $E_{\mathcal{R}}(v)$ on $\widetilde{\mathbf{U}}$ by setting

$$
E_{\mathcal{R}}(v)(z):= \begin{cases}v(\mathcal{R}(z)), & \text { if } z \in A\left(1-\epsilon_{2}, 1\right), \\ 0, & \text { if } z \in \partial \mathbb{D}, \\ v(z), & \text { if } z \in \widetilde{\mathbf{U}} \backslash \overline{\mathbb{D}} .\end{cases}
$$

It is easy to see for every $z \in \mathbf{U}$, we have

$$
E_{\widetilde{\mathcal{R}}}(u)(z)=E_{\mathcal{R}}(v)(h(z)) .
$$

By Eq. 3.29, $\left.v\right|_{A\left(1,1+\epsilon_{1}\right)}$ belongs to the class $\dot{W}^{1,2}\left(A\left(1,1+\epsilon_{1}\right)\right)$. To simplify the notation, we still denote $\left.v\right|_{A\left(1,1+\epsilon_{1}\right)}$ by $v$. By facts that $\mathcal{R}\left(A\left(1-\epsilon_{2}, 1+\epsilon_{1}\right)\right)=A\left(1-\epsilon_{2}, 1+\epsilon_{1}\right)$, $\dot{W}^{1,2}\left(A\left(1-\epsilon_{2}, 1+\epsilon_{1}\right)\right)=W^{1,2}\left(A\left(1-\epsilon_{2}, 1+\epsilon_{1}\right)\right)$ and $C^{\infty}\left(\mathbb{R}^{2}\right) \cap W^{1,2}\left(A\left(1-\epsilon_{2}, 1+\epsilon_{1}\right)\right)$ is dense in $W^{1,2}\left(A\left(1-\epsilon_{2}, 1+\epsilon_{1}\right)\right)$. With a similar argument to the inequality (3.17), we obtain $E_{\mathcal{R}}(v) \in \dot{W}^{1,2}(\widetilde{\mathbf{U}})$ with

$$
\begin{aligned}
\int_{A\left(1-\epsilon_{2}, 1+\epsilon_{1}\right)} & \left|D E_{\mathcal{R}}(v)(z)\right|^{2} d z \\
\leq & C \int_{A\left(1,1+\epsilon_{1}\right)}|D v(z)|^{2} d z \\
\leq & C \int_{h(B(0, R) \backslash \bar{\Omega})}|D v(z)|^{2} d z
\end{aligned}
$$

for a positive constant $C$ that is independent of $u$. Then, with a similar argument to the inequality (3.18), we obtain the inequality

$$
\begin{aligned}
& \left(\int_{\mathcal{A}}\left|D E_{\mathcal{R}}(u)(z)\right|^{\frac{2 p}{p+1}} d z\right)^{\frac{p+1}{2 p}} \\
& \quad \leq C\left(\int_{A\left(1-\epsilon_{2}, 1\right)}\left|D E_{\mathcal{R}}(v)(z)\right|^{2} d z\right)^{\frac{1}{2}}
\end{aligned}
$$

for a positive constant $C$ that is independent of $u$.

Finally, by combining inequalities (3.29), (3.31) and (3.32), we obtain the desired inequality (3.28).

\section{Sharpness and applications of Theorem 1.2}

\subsection{Sharpness of Theorem 1.2}

In this section, we discuss the sharpness of Theorem 1.2. Let $\Omega=\Omega_{t^{s}}^{i} \subset \mathbb{R}^{2}$ be a polynomial inward cuspidal domain with the degree $1<s<\infty$ such that there exists a homeomorphism of finite distortion $h: \mathbb{R}^{2} \stackrel{\text { onto }}{\longrightarrow} \mathbb{R}^{2}$ with $h(\Omega)=\mathbb{D}$, and the distortion function satisfies the integral condition (3.1). It is easy to see $\mathbb{R}^{2} \backslash \overline{\Omega_{t^{s}}^{i}}$ has a same Sobolev 
extension property with the polynomial outward cuspidal domain $\Omega_{t^{s}}^{o}$. By combining Propositions 2.2 and 2.3, we obtain that we cannot obtain a better Sobolev extension result than $\Omega$ is a Sobolev $\left(\frac{2 p}{p-1}, \frac{2 q}{q+1}\right)$-extension domain and $\mathbb{R}^{2} \backslash \overline{\Omega_{t^{s}}^{i}}$ is a Sobolev $\left(\frac{2 q}{q-1}, \frac{2 p}{p+1}\right)$-extension domain. It shows the sharpness of Theorem 1.2.

\subsection{A new proof of the necessity of Proposition 2.3}

The necessity claim in Proposition 2.3 is proved by combing Theorem 1.2 and Proposition 2.2 .

\subsection{An application of Theorem 1.2}

In this section, we prove Theorem 1.3, which can be regarded as an application of Theorem 1.2 .

Proof of Theorem 1.3 By [17, Theorem 1.6], there exists a positive constant $0<\beta<2$ such that for every homeomorphism of finite distortion $h: \mathbb{R}^{2} \stackrel{\text { onto }}{\longrightarrow} \mathbb{R}^{2}$ with $\exp \left(\lambda K_{h}\right) \in$ $L_{\text {loc }}^{1}\left(\mathbb{R}^{2}\right)$ with $\lambda>0$, we have $K_{h^{-1}} \in L_{\text {loc }}^{p_{\lambda}}\left(\mathbb{R}^{2}\right)$ with $p_{\lambda}=\beta \lambda$. Let $h: \mathbb{R}^{2} \stackrel{\text { onto }}{\longrightarrow} \mathbb{R}^{2}$ be a homeomorphism of finite distortion with $\exp \left(\lambda K_{h}\right) \in L_{\text {loc }}^{1}\left(\mathbb{R}^{2}\right)$ for some large enough $\lambda>0$ with $p_{\lambda}=\beta \lambda>1$. Then by Theorem $1.2, \Omega:=h^{-1}(\mathbb{D})$ is a $L^{p_{\lambda}}$-quasidisk and is a Sobolev $\left(\frac{2 p_{\lambda}}{p_{\lambda}-1}, \frac{2 p_{\lambda}}{p_{\lambda}+1}\right)$-extension domain.

Funding Open access funding provided by University of Jyväskylä (JYU).

Data Availability Statement The author declares that the data supporting the findings of this study are available within the article.

Open Access This article is licensed under a Creative Commons Attribution 4.0 International License, which permits use, sharing, adaptation, distribution and reproduction in any medium or format, as long as you give appropriate credit to the original author(s) and the source, provide a link to the Creative Commons licence, and indicate if changes were made. The images or other third party material in this article are included in the article's Creative Commons licence, unless indicated otherwise in a credit line to the material. If material is not included in the article's Creative Commons licence and your intended use is not permitted by statutory regulation or exceeds the permitted use, you will need to obtain permission directly from the copyright holder. To view a copy of this licence, visit http://creativecommons.org/licenses/by/4.0/.

\section{References}

1. Ahlfors, L.V.: Quasiconformal reflections. Acta Math. 109, 291-301 (1963)

2. Astala, K., Iwaniec, T., Martin, G.: Elliptic Partial Differential Equations and Quasiconformal Mappings in the Plane. Princeton University Press, Princeton (2009)

3. Burenkov, V.I.: On a method of extension of differentiable functions. Trudy Mat. Inst. Steklov 140, 27-67 (1976). (Russian); English transl. in Proc. Steklov Inst. Math., American Mathematical Society, Providence, Rhode Island 140 (1979, issue 1)

4. Burenkov, V.I.: Extension of functions preserving certain smoothness and compactness of embeddings for spaces of differentiable functions. Trudy Math. Inst. Steklov 248, 74-85 (2005). (Russian). English transl. in Proc. Steklov Inst. Math., American Mathematical Society, Providence, Rhode Island, 248, 2005

5. Evans, L.C., Gariepy, R.F.: Measure Theory and Fine Properties of Functions, Studies in Advanced Mathematics. CRC Press, Boca Raton (1992) 
6. Fain, B.L.: Extension of functions from Sobolev spaces for irregular domains preserving the index of smoothness. (Russian) Dokl Akad. Nauk SSSR 285(2), 296-301 (1985)

7. Federer, H.: Geometric Measure Theory, Die Grundlehren Der Mathematischen Wissenschaften, 2nd edn, Band, vol. 153. Springer, New York (1996)

8. Gehring, F.W.: Univalent functions and the Schwarzian derivative. Comment. Math Helv. 52, 561-572 (1977)

9. Gehring, F.W.: Characteristic Properties of Quasidisks Séminaire De Mathématiques Supérieures [Seminar on Higher Mathematics], vol. 84. Presses de l'Université de Montréal, Montreal (1982)

10. Gehring, F.W.: Uniform domains and the ubiquitous quasidisk. Jber Deutsche Math. Verein. 89, 88-103 (1987)

11. Gehring, F.W., Hag, K.: Remorks on uniform and quasiconformal extension domains. Complex Var. Elliptic Equ. 9, 175-188 (1987)

12. Gehring, F.W., Hag, K.: Reflections on Reflections in Quasidisks, Papers on Analysis, 81-90, Rep. Univ. Jyväskylä Dep. Math Stat., vol. 83. Univ. Jyväskylä, Jyväskylä (2001)

13. Gehring, F.W., Hag, K.: The Ubiquitous Quasidisk. With Contributions by Ole Jacob Broch Mathematical Surveys and Monographs, vol. 184. American Mathematical Society, Providence, RI (2012)

14. Gilbarg, D., Trudinger, N.S. Elliptic Partial Differential Equations of Second Order, 2nd edn. Springer, Berlin (1983)

15. Gol'dshtein, V.M., Sitnikov, V.N.: Continuation of functions of the class $w_{p}^{1}$ across hölder boundaries. (Russian) Imbedding theorems and their applications, 31-43, Trudy Sem. S. L. Soboleva, No. 1 Akad. Nauk SSSR Sibirsk. Otdel., Inst, Mat., Novosibirsk (1982)

16. Guo, C., Koakela, P., Takkinen, J.: Generalized quasidisks and conformality. Publ. Mat. 58(1), 193-212 (2014)

17. Hencl, S., Koskela, P.: Regularity of the inverse of a planar Sobolev homeomorphism. Arch. Ration. Mech Anal. 180(1), 75-95 (2006)

18. Hencl, S., Koskela, P.: Lectures on Mappings of Finite Distortion Lecture Notes in Mathematics, vol. 2096. Springer, Cham (2014)

19. Herron, D.A., Koskela, P.: Uniform, Sobolev extension and quasiconformal circle domains. J. Anal. Math. 57, 172-202 (1991)

20. Hurri, R.: Poincaré domains in $\mathbb{R}^{n}$. Ann. Acad. Sci. Fenn. Ser. A I Math. Diss. 71, 1-42 (1988)

21. Iwaniec, T., Martin, G.: Geometric Function Theory and Non-linear Analysis, Oxford Mathematical Monographs. Oxford University Press, Oxford (2001)

22. Iwaniec, T., Onninen, J., Zhu, Z.: Singularities in $\mathscr{P} L^{p}$-quasidisks. Annales Fennici Mathematici 46(2), 1053-1069 (2021)

23. Iwaniec, T., Onninen, J., Zhu, Z.: Bi-Sobolev boundary singularities, submitted (2020)

24. Jones, P.: Quasiconformal mappings and extendability of functions in Sobolev spaces. Acta Math. 147(12), 71-88 (1981)

25. Koskela, P., Takkinen, J.: Mappings of finite distortion: formation of cusps. Publ. Mat. 51(1), 223-242 (2007)

26. Koskela, P., Takkinen, J.: Mappings of finite distortion: formation of cusps. III. Acta Math. Sin. (Engl. Ser.) 26(5), 817-824 (2010)

27. Koskela, P., Takkinen, J.: A note to Mappings of finite distortion: formation of cusps II. Conform Geom. Dyn. 14, 184-189 (2010)

28. Koskela, P., Ukhlov, A., Zhu, Z.: The volume of the boundary of Sobolev $(p, q)$-extension domains. Preprint (2020)

29. Koskela, P., Zhu, Z.: Sobolev extension via reflections, arXiv:1812.09037 (2020)

30. Martio, O., Sarvas, J.: Injectivity theorems in plane and space. Ann. Acad. Sci. Fenn. 4, 383-401 (Unknown Month 1978)

31. Maz'ya, V.G.: Sobolev Spaces. Translated from the Russian by T.O. Shaposhnikova. Springer Series in Soviet Mathematics. Springer, Berlin (1985)

32. Maz'ya, V.G., Poborchi, S.V.: On extension of functions in Sobolev classes to the exterior of a domain with the vertex of a peak on the boundary. Dokl. Akad. Nauk SSSR 275:5 (1984) 1066-1069 (Russian). English translation: Soviet Math. 29, 361-364 (1984)

33. Maz'ya, V.G., Poborchi, S.V.: Extension of functions in Sobolev classes to the exterior of a domain with the vertex of a peak on the boundary I. Czech. Math. Journ. 36(111), 634-661 (1986). (Russian)

34. Maz'ya, V.G., Poborchi, S.V.: Extension of functions in Sobolev classes to the exterior of a domain with the vertex of a peak on the boundary II. Czech. Math. Journ. 37(112), 128-150 (1987). (Russian)

35. Maz'ya, V.G., Poborchi, S.V.: Differentiable functions on bad domains. World Scientific Publishing Co. Inc, River Edge (1997) 
36. Ukhlov, A.D.: On mappings that generate embeddings of Sobolev spaces, (Russian) Sibirsk. Mat. Zh. 34(1), 185-192 (1993). Translation in Siberian Math. J. 34(1993), no 1 165-171

37. Ziemer, W.P.: Weakly differentiable functions. Sobolev Spaces and Functions of Bounded Variation Graduate Texts in Mathematics, vol. 120. Springer, New York (1989)

Publisher's Note Springer Nature remains neutral with regard to jurisdictional claims in published maps and institutional affiliations. 